\title{
Statistical Properties of the Mid and Far IR Emission in Mixed Morphology (E+S) Pairs of Galaxies.
}

\author{
H. M. Hernández Toledo and D. Dultzin-Hacyan \\ Instituto de Astronomía, UNAM Apdo. 70-264, México, D. F., 04510 \\ J. W. Sulentic \\ Department of Physics and Astronomy, University of Alabama, \\ Tuscaloosa, AL 35487
}

\section{Introduction}

We report the results of a detailed statistical analysis of the Mid and Far IR (MIR/FIR) emission properties in a mixed morphology (E+S) sample of galaxy pairs from the Karachentsev (1972) Catalogue. The sample is large and diverse enough ( $\sim 130$ pairs) to permit discrimination of pairs by morphological types and interaction classes. It samples a large enough volume to enable a non-parametric estimate of a Far-Infrared Luminosity Function (FIRLF). We find average factors of 3 and 5 enhancement in FIR and $25 \mu \mathrm{m}$ luminosities of the late-type pair components relative to an isolated galaxy control sample from Karachentseva (1973) Catalogue. This is interpreted as the MIR/FIR signature of the link between interaction and star formation. Although the spirals in $(\mathrm{E}+\mathrm{S})$ pairs fail to show a trend towards higher FIR luminosity with decreasing companion separation, a high-order comparison that takes into account information on the interaction classes, morphological types and the $\mathrm{H}$ I content suggests that:

- a clear correlation between the IR luminosities and interaction classes is present suggesting that a subsample of the $(\mathrm{E}+\mathrm{S})$ pairs is more closely related to recent events of star formation,

- the ratio of present to past star formation (as measured by the IR luminosities scaled to $L_{B}$ luminosity), increases measurably along the sequence $\mathrm{Sa}-\mathrm{Sc}$ in paired spirals,

- no $\mathrm{H}$ I depletion in the star formation-enhanced spirals in mixed pairs is found, and

- the high mass $\left(M \geq 10 M_{\odot}\right)$ star formation rate (SFR) in paired Sc spirals is higher than that of the isolated Sc's by a factor $\sim 2-3$.

We consider both possible bias and dynamical explanations for the results obtained. Our statistical analysis is based on 1) a new re-reduction of the IR fluxes for all the mixed and isolated galaxies by means of the SCANPI/HIRES facilities at IPAC. This improves statistics for the 60 and 100 micron comparisons and provide enough detections at 25 microns to allow this band to be used as 
an additional statistical variable. 2) A more uniform estimation of upper limits which are at the basis of our interpretation through non-parametric statistical methods.

\section{Discussion}

One of the big surprises that emerged from the Catalogue of Isolated Pairs of Galaxies (Karachentsev 1972) was the large number of (E+S) pairs. Such a large number is surprising if local environment strongly influences galaxy morphology. This was the theoretical motivation for the belief that most pairs had morphologically concordant components. This general belief led some (Faber \& Gallagher 1979; Noerdlinger 1979) to argue that the (E+S) pairs should have largely discordant or accordant optical alignments. Perhaps a fraction of the $\mathrm{E}+\mathrm{S}$ pairs are the product of secular evolution from (partially merged) higher order multiplets or originally morphologically-concordant pairs (Rampazzo \& Sulentic (1992); Wiren et al. 1996). However the large representation found for mixed types and the evidence for a statistically significant optical enhancement (Hernández-Toledo et al. 1999) as well as the MIR/FIR enhancement reported here, suggest that an important fraction of them can be considered as physical binaries. This is a puzzle to the theories of galaxy formation: how can mixed pairs exist at all in significant numbers when local environmental conditions are thought to dictate galaxy morphology?

Acknowledgments. Those of us fortunate enough to have been able to participate in this best meeting on Small Galaxy Groups held in Turku, Finland, owe a great debt to its organizers. We gratefully acknowledge the support of all of them.

\section{References}

Faber, S. M., Gallagher, J. S. 1979, ARA\&A, 17, 135.

Hernández Toledo, H. M., Dultzin-Hacyan, D., González , J. J., and Sulentic, J. W. 1999, AJ, 118, 108.

Karachentseva, V. E., 1973, Comm. Spec. Ap. Obs. USSR, 8, 1.

Karachentsev, I. D. 1972, Comm. Spec. Ap. Obs. USSR. 7, 1.

Noerdlinger, P. D. 1979, ApJ, 229, 877.

Rampazzo, R., and Sulentic, J. W. 1992, A\&A, 259, 43.

Wiren, S., Zheng, J., Valtonen, M. J., Chernin, A. D. 1996, AJ, 111160. 